\title{
El uso de fluidos supercríticos en la industria de aceites alimentarios
}

\author{
Por M. Esquível y M.G. Bernardo-Gil \\ Departamento de Ingeniería Química. Instituto Superior Técnico. Av. Robisco Pais. \\ 1096 Lisboa Codex. PORTUGAL.
}

\section{RESUMEN}

El uso de fluidos supercríticos en la industria de aceites alimentarios.

Se han realizado experiencias de extracción de aceite de orujo con dióxido de carbono a presiones de 12 y $15 \mathrm{MPa}$ y a las temperaturas de $308.15,313.15$ y $318.15 \mathrm{~K}$.

Las pruebas efectuadas permiten concluir que en esta gama de presiones y a la temperatura de $313.15 \mathrm{~K}$ no existe extracción selectiva de los ácidos grasos y que se obtienen rendimientos elevados en relativamente poco tiempo.

PALABRAS-CLAVE: Aceite comestible - Acido graso - Extracción Fluido supercrítico.

\section{SUMMARY}

The use of supercritical fluids en the edible oil industry.

Residual olive oil extraction experiments were made with carbon dioxide under pressures of 12 and $15 \mathrm{MPa}$ and $308.15,313.15$ and $318.15 \mathrm{~K}$ of temperature.

We may conclude through the experiments, that under these pressures and temperature of $313.15 \mathrm{~K}$ there is no selected extraction of fatty acids, and higher recovery rates can be obtained in a short period of time.

KEY-WORDS: Edible oil - Extraction - Fatty acid - Supercritical fluid.

\section{INTRODUCCION}

Un fluido supercrítico es un gas o líquido en condiciones de presión y temperatura a las de su punto crítico. Se considera punto crítico aquél en el que las fases líquida y vapor se vuelven indistintas, esto es, la fase crítica, determinada normalmente por los parámetros: presión crítica, temperatura crítica y densidad crítica.

En esta fase, el fluido tiene propiedades típicas que lo hacen especialmente indicado como solvente de extracción, así:

- El hecho de poseer viscosidades próximas a las del gas, aumenta su poder de difusión.

- Densidades próximas a las del líquido, favorece la interacción entre las moléculas de solvente y soluto.

- Con pequeñas variaciones de presión y temperatura se puede variar la densidad del fluido controlando así su poder solvente.

- La separación del solvente puede ser hecha sencillamente por variación de la presión y/o temperatura.
- Poseer coeficientes de difusión próximos a los del líquido, lo que facilita el fenómeno de transporte.

Los fluidos utilizados como solventes de extracción supercrítico son el dióxido de carbono, propano, agua, amoníaco, hexano, etileno, tolueno y el óxido nitroso.

De todos los solventes referidos, el más utilizado es sin duda alguna el dióxido de carbono, a causa de los bajos valores de sus parámetros críticos (especialmente la temperatura, que permite su utilización en extracciones con productos volátiles o que se alteran químicamente a altas temperaturas), ser químicamente inerte, no tóxico (lo que lo hace adecuado para la industria alimentaria), no corrosivo y barato.

Se ha comprobado que el consumo de dióxido de carbono es mucho más alto que el de otros solventes habituales para el mismo grado de extracción, aunque este hecho pueda ser mejorado con la recirculación del solvente. Además, ya que el dióxido de carbono es apolar será de esperar que sea buen solvente para compuestos apolares, razón por la cual muchas veces se utilizan modificadores (como por ejemplo en la extracción de la cafeína del café). A pesar de los dos inconvenientes apuntados, sigue siendo el solvente supercrítico por excelencia.

\section{Extracción Supercrítica de Aceites Alimentarios}

Las buenas potencialidades de esta nueva tecnología ha despertado el interés de los investigadores. Varios autores han escrito sobre los conceptos generales de la extracción supercrítica (1) (4) (5) (12) (19) (25) (26) (27) (28) (29).

Esta tecnología tiene su principal aplicación en la extracción de aceites alimentarios (9) (10) (21) (22) (31) (33) (34), como son el aceite de cacahuete (18), aceite de soja (13) (16) (23), aceite de maíz (7) (8) y aceite de colza (11) (14).

En el campo de los aceites comestibles también se han llevado a cabo con éxito trabajos de investigación, referentes a la concentración de tocoferoles a partir de los subproductos de refinación (30), y en la refinación de aceites vegetales destaca la separación de los triglicéridos de los ácidos grasos libres (34) (15). 
Se han determinado solubilidades de algunos ésteres de ácidos grasos en solventes supercríticos (20) (24) (35). Más recientemente han apareceido trabajos de investigación en el campo de la extracción del aceite de oliva (17), en la refinación del aceite de oliva utilizando columna de relleno (3), y en la desacidificación del aceite de oliva recurriendo a condiciones experimentales en las que el solvente supercrítico presenta una gran selectividad (6).

Los buenos resultados obtenidos han llevado a un interés creciente en este campo. El paso de la extracción de aceites a escala industrial se encuentra aún en fase de estudio. Las plantas industriales existentes actualmente son las utilizadas para la descafeinación del café, extracción del lúpulo, reducción de nicotina del tabaco y obtención de extractos de ciertas especias con alto valor añadido.

\section{El Orujo de Aceituna}

Durante el proceso de fabricación del aceite de oliva, la aceituna es triturada obteniéndose una pulpa (orujo graso de aceituna) que es prensada para la extracción de la materia grasa. El aceite de oliva es obtenido después de una centrifugación del producto de la prensa.

La torta que se obtiene por prensa -orujo graso- contiene cerca del $8-15 \%$ de aceite que puede ser extraído con hexano (el aceite de orujo). Este aceite tiene una acidez muy elevada (cerca de 30 grados) por lo que tiene que ser sometido a operaciones de neutralización, decoloración y desodorización antes de poder ser consumido.
Se ha llevado a cabo la extracción de aceite de orujo con dióxido de carbono supercritico estudiando la variación de la solubilidad del aceite con la presión y la temperatura, con la finalidad de determinar las potencialidades de la extracción supercrítica como alternativa al proceso clásico. Se ha determinado la composición en ácidos grasos del aceite, obtenido por extracción supercrítica comparándola con la del extraido con hexano.

\section{EQUIPAMIENTO}

En la figura 1 se presenta el diagrama de la planta de extracción instalada en el Instituto Superior Técnico de Lisboa.

El dióxido de carbono a la presión de la botella (aproximadamente $5 \mathrm{MPa}$ ) pasa por un baño muy frío a la temperatura de $273.15 \mathrm{~K}$, para garantizar su licuefacción total, y es llevado a una bomba tipo "air-driven" que lo presuriza hasta la presión deseada.

En "by-pass" a la bomba, están montadas una válvula de seguridad V1 y una válvula del tipo "back-pressure regulator" V2.

El solvente fundido pasa de forma contínua por un intercambiador de calor donde es pre-calentado hasta la temperatura de trabajo y de ahí a un extractor tubular de acero inoxidable que contiene el orujo graso de aceituna y que se encuentra inmerso en un baño a temperatura controlada.

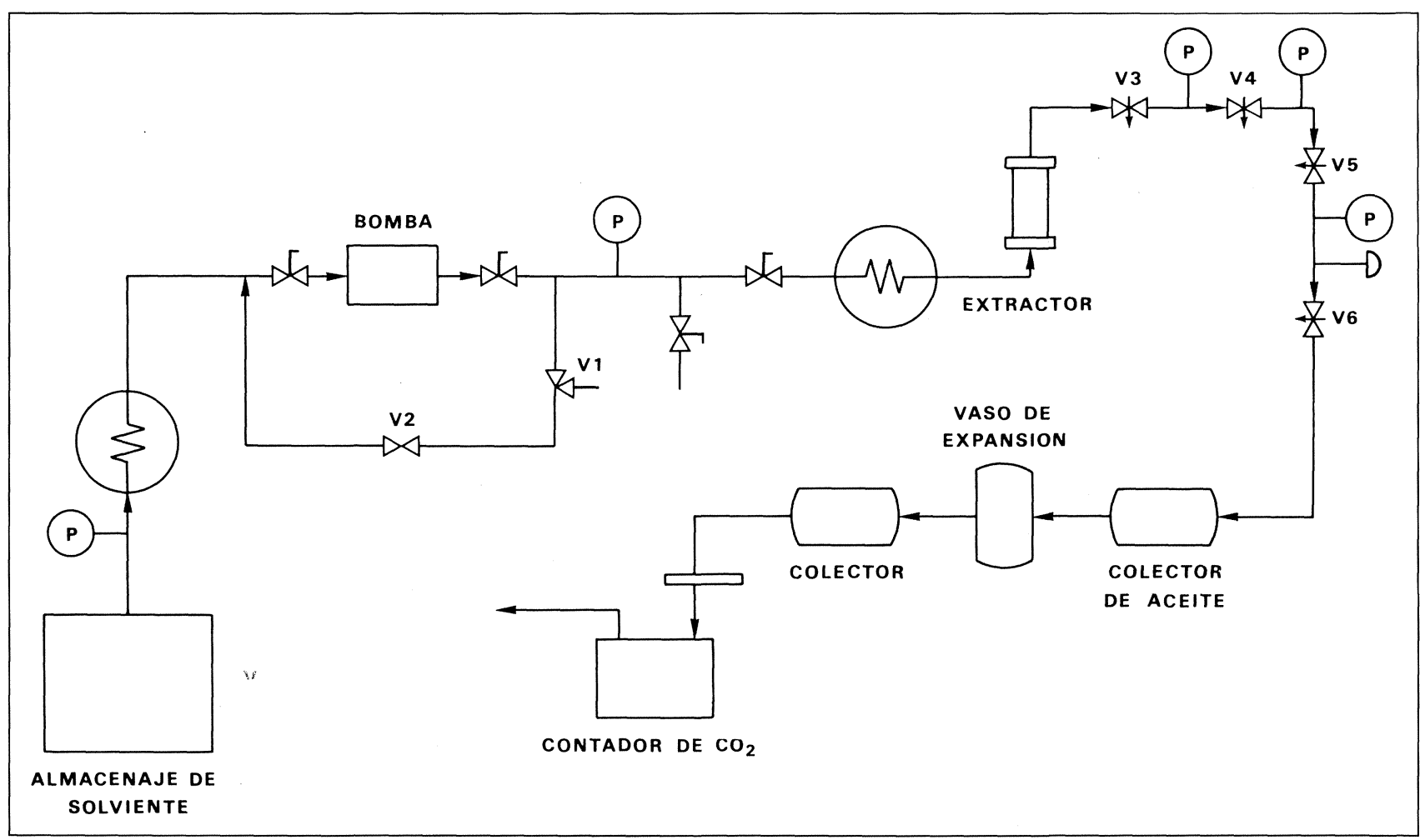




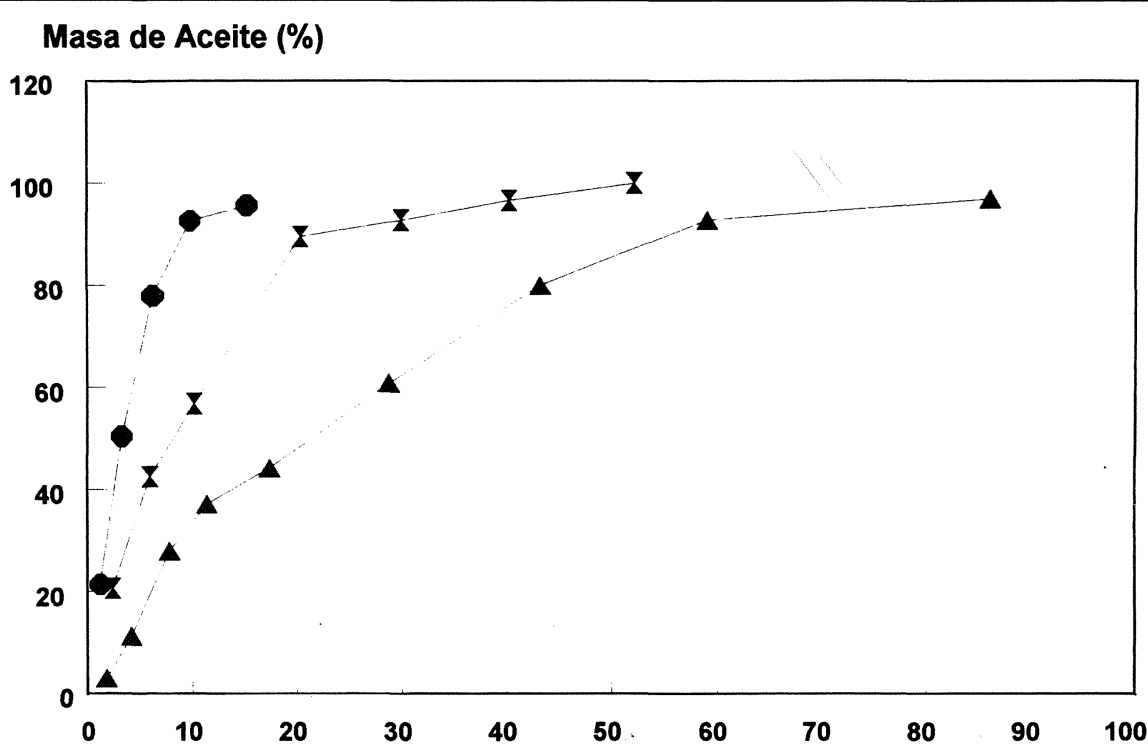

Tiempo de Extraccion (s) $\times 10^{-3}$

$$
\triangle \mathrm{P}=12 \mathrm{MPa} \quad \mathrm{P}=15 \mathrm{MPa} \quad \mathrm{P}=18 \mathrm{MPa}
$$

A la salida del extractor es donde se controla el caudal de solvente, mediante una válvula de aguja. La presión del dióxido de carbono es reducida en este punto en varias etapas, hasta la presión atmosférica (V3, V4, V5), recuperándose en el primer colector el aceite de orujo. El agua y los componentes volátiles son retirados en el segundo colector a una temperatura de $203.15 \mathrm{~K}$.

En la última fase del proceso se mide la temperatura del gas, siendo determinado con un contador de gas la cantidad de dióxido de carbono utilizado.

\section{RESULTADOS Y DISCUSION}

En la figura 2 se presentan las curvas de extracción de la cantidad de aceite de orujo (\% w/w) en función del tiempo de extracción (s) para las presiones de 12, 15 y 18 $\mathrm{MPa}$, a la temperatura de $318.15 \mathrm{~K}$ y velocidad superficial del gas de $0.06 \times 10^{-2} \mathrm{~m} / \mathrm{s}$. Las figuras presentadas son acumulativas y se verifica que para tiempos superiores a 20000 s las cantidades extraídas son muy pequeñas, no continuándose la experiencia a $18 \mathrm{MPa}$ para tiempos superiores.

En la figura 3 se presentan las curvas de extracción del aceite de orujo de aceitunas para una presión de $15 \mathrm{MPa}$ a temperaturas de $308.15,313.15$ y $318.15 \mathrm{~K}$.

Como se puede comprobar, la solubilidad del aceite de orujo disminuye con el aumento de la temperatura. Este factor ha sido también ya verificado por otros autores (14) (24) que imputan este comportamiento a la disminución del valor de la densidad del fluido con la temperatura en la gama de presiones estudiada (la disminución de la densidad del dióxido de carbono hace bajar su poder solvente).

En la tabla I se presenta la composición porcentual en ácidos grasos de las fracciones obtenidas durante la extracción supercrítica a $12 \mathrm{MPa}$ y $313.15 \mathrm{~K}$, y la del aceite de orujo extraído con hexano, para su comparación. Se ha observado que el aceite de orujo obtenido de $\mathrm{CO}_{2}$ supercrítico tiene contenidos ligeramente más elevados en ácido palmítico y en ácido palmitoleico y contenidos ligeramente inferiores en ácido oleico, aunque las diferencias no sean significativas.

Como se puede observar, la extracción es extremadamente rápida, obteniéndose porcentajes bastante elevados con bajos tiempos de extracción. En esta temperatura y en esta gama de presiones la solubilidad aumenta con la presión y disminuye con el aumento de temperatura.

La composición porcentual en ácidos grasos durante la extracción permanece aproximadamente constante y próxima a las gamas para el aceite orujo obtenido por el proceso clásico de extracción con hexano. En la gama de temperaturas estudiada, a medida que se aumenta la presión, el poder solvente del $\mathrm{CO}_{2}$ aumenta, pero la selectividad disminuye. De ahí que en principio, la extracción preferencial en esta presión sea poco probable que ocurra a presiones más elevadas.

Las principales ventajas de la utilización del dióxido de carbono supercrítico como solvente de extracción de aceites son básicamente: su disponibilidad, bajo costo, no toxicidad, no contamina el ambiente y puede ser fácilmen- 


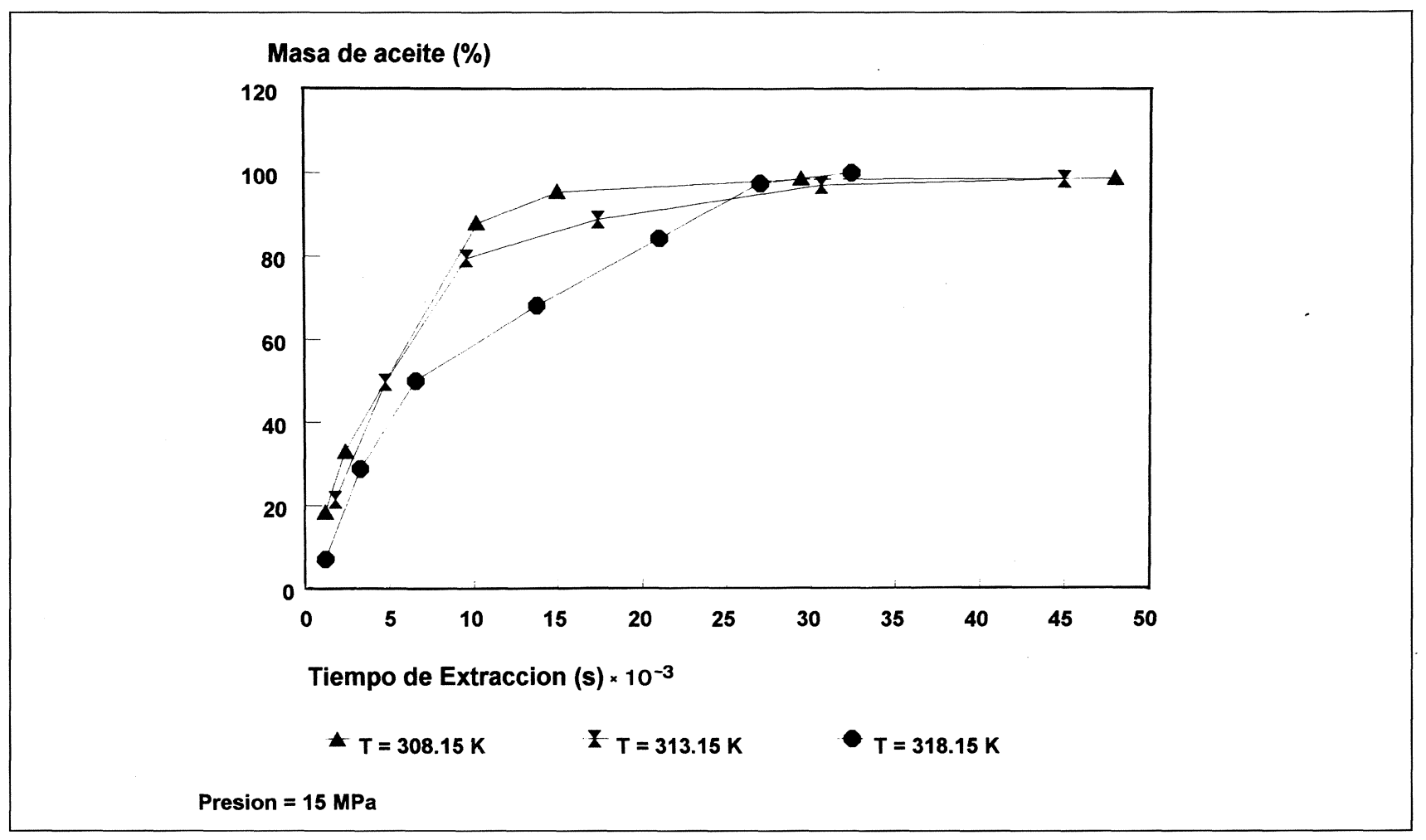

Figura 3

Extracción de aceite de orujo en función de la temperatura

Tabla I

Composición en ácidos grasos (\% w/w) de aceite de orujo extraído con $\mathrm{CO}_{2}$ supercrítico a $12 \mathrm{MPa}$ y $313.15 \mathrm{~K}$

\begin{tabular}{|c|c|c|c|c|c|c|c|}
\hline \multirow{3}{*}{$\begin{array}{l}\text { ÁcIDOS } \\
\text { GRASOS }\end{array}$} & \multicolumn{7}{|c|}{ ACEITE DE ORUJO } \\
\hline & \multirow{2}{*}{$\begin{array}{l}\text { Extracción } \\
\text { con Hexano }\end{array}$} & \multicolumn{6}{|c|}{ ESC en el tiempo (h) } \\
\hline & & 0.5 & 1.2 & 3.2 & 4.8 & 8.0 & 12.0 \\
\hline Láurico & & 4.39 & 0.54 & 0.20 & 0.13 & 0.09 & 0.08 \\
\hline Mirístico & 0.01 & - & - & 0.05 & * & * & 0.17 \\
\hline Palmítico & 11.98 & 13.1 & 12.1 & 14.3 & 14.5 & 15.3 & 15.2 \\
\hline Palmitoleico & 1.82 & 2.24 & 3.22 & 2.14 & 2.18 & 2.48 & 2.32 \\
\hline Heptadecanoico & 0.35 & 1.11 & 1.15 & 0.18 & * & * & 0.24 \\
\hline Heptadecenoico & 0.57 & * & 0.31 & 0.31 & * & * & 0.41 \\
\hline Esteárico & 2.39 & 2.89 & 1.45 & 1.74 & 2.13 & 1.37 & 2.01 \\
\hline Oleico & 72.3 & 69.7 & 69.1 & 70.2 & 70.1 & 73.1 & 73.3 \\
\hline Linoleico & 8.09 & 6.62 & 6.29 & 7.57 & 9.21 & 7.62 & 4.15 \\
\hline Araquídico & 1.76 & - & 0.55 & 1.70 & * & * & 0.81 \\
\hline Linolenico & 0.68 & & * & 1.27 & * & * & 0.65 \\
\hline Behénico & * & - & * & * & * & * & * \\
\hline Otros & 0.11 & - & 5.30 & 0.36 & 1.74 & 0.01 & 0.66 \\
\hline
\end{tabular}

(*) Análisis no concluyente. 
te separado del aceite extraído por simple descompresión de la mezcla solvente-soluto.

Se está efectuando la caracterización de los aceites de orujo obtenidos con diferentes condiciones de presión y temperatura. En este momento están en curso en el Instituto Superior Técnico trabajos de investigación en el campo de la extracción supercrítica aplicada a la extracción de aceites alimentarios, con la finalidad de estudiar la posibilidad de refinar, desacidificar y desodorizar el aceite de oliva y el aceite de orujo por medio de las variaciones de las condiciones de extracción.

\section{AGRADECIMIENTOS}

Las autoras dan las gracias al INIC, JNICT, V. Guedes, Simão e $C^{a}$ y al Dr. M.B. King de la Universidad de Birmingham, por el apoyo concedido en la realización de este trabajo de investigación.

\section{BIBLIOGRAFÍA}

1. Artozoul, J. y Bernard, M. (1987).- "Extraction à l'Anhydride Carbonique".Parfums, Cosmetiques, Aromes 75, 89-94.

2. Bernardo-Gil, M.G. y Esquível, M. (1991).- "Extracção de Óleo de Bagaço de Azeitona com $\mathrm{CO}_{2}$ Supercrítico".- $12 \mathrm{r}$ Encontro da Sociedade Portuguesa de Química, Coimbra, Portugal, 143-146.

3. Bondioli, P., Mariani, C., Lanzani, A., Fedeli, E., Mossa, A. y Muller, A. (1992).- "Lampante Olive Oil Refining with $\mathrm{SC} \mathrm{CO}_{2}$ ".- J. Am. Oil Chemists' Soc. 69, 477-480.

4. Bott, T.R. (1982).- "Fundamentals of Carbon Dioxide in Solvent Extraction".- Chem. Ind. 12, 394-396.

5. Brogle, H. (1982).- " $\mathrm{CO}_{2}$ in Solvent Extraction".-- Chem. Ind. 12, 385-390.

6. Brunetti, L., Daghetta, A., Fedeli, E., Kikic, I., y Zanderighi, L. (1989)."Deacidification of Olive Oils by Supercritical Carbon Dioxide".- J. Am. Oil Chemists' Soc. 66, 209-217.

7. Chien, J.T., Hoff, J.E. y Lee, M.J. (1990).- "Oil Extraction of Dried Ground Corn with Ethanol".- Chem. Eng. Journal 43, B103-B113.

8. Christianson, D.D. Friedrich, J.P., List, G.R., Warner, K., Bragley, E.B. Stringfeltow, A.C. y Inglett, G.E. (1984).- "Supercritical Fluid Extraction of Dry-Milled Corn Germ with Carbon Dioxide".- J. Food Sci. 49, 229232.

9. Dakovic, S., Turkulov, J., Dimic, E. (1989).- "The Quality of Vegetable Oils Got by Extraction with $\mathrm{CO}_{2} "$.- Fat Sci. Technol. 91, 116-119.

10. Eggers, R. (1985).- "High Pressure Extraction of Oil Seed".- J. Am. Oil Chemists' Soc. 62, 1222-1230.

11. Eggers, R. y Sievers, U. (1989).- "Processing of Oilseed with Supercritical Carbon Dioxide".- J. Chem. Eng. Jpn. 22, 641-649.

12. Eisenbach, W.O. (1987).- "Supercritical Carbon Dioxide as an Extraction Agent".- Nato ASI Ser., Ser C 206 (Carbon Diox Source Carbon: Bioch. Chem. Uses), 371-388.

13. Eldridge, A.C., Friedrich, J.P., Warner, K. y Wolek, W.F.K. (1986)."Preparation and Evaluation of Supercritical Carbon Dioxide Defatted Soybean Flakes".- J. Food Sci. 51, 584-587.

14. Fattori, M., Bulley, N.R. y Meisen, A. (1988).- "Carbon Dioxide Extraction of Canola Seed: Oil Solubility and Effect of Seed Treatment".- J. Am. Oil Chemists' Soc. 65, 968-974.
15. Friedrich, J.P. y List, G.R. (1982).- "Characterization of Soybean Oil Extraction by Supercritical Carbon Dioxide and Hexane".- J. Agric. Food Chem. 30, 193-193.

16. Friedrich, J.P., List, G.R. y Heakin, A.J. (1982).- "Petroleum-Free Extraction of Oil from Soybeans with Supercritical $\mathrm{CO}_{2}{ }^{\prime \prime} .-\mathrm{J}$. Am. Oil Chemists' Soc. 59, 288-292.

17. Giovacchino, L. Di, Solinas, M., Brandani, V., Del Re, G. y Di Giacomo G. (1989).- "Risultati delle Prove Preliminari Di Estrazione Dell'Olio Dalle Olive Con $\mathrm{CO}_{2}$ Supercritica".- Ind. Aliment. 28, 925-928.

18. Goodrum, J.W. y Kilgo, M.B. (1987).- "Peanut Oil Extraction Using Compressed $\mathrm{CO}_{2}$ ".- Energy Agric. 6, 265-271.

19. Hawthorne, S.B. (1990).- "Analytical-Scale Supercritical Fluid Extraction".- Anal. Chem. 62, 663A-642A

20. Ikushima, Y., Saito, N. y Goto, T. (1989).- "Selective Extraction of Oleic, and Linolenic Acid Methyl Esters from Their Mixture with Supercritical Carbon Dioxide-Entrainer Systems and a Correlation of the Extraction Efficiency with a Solubility Parameter".- Ind. Eng. Chem. Res. 28, 1364-1369.

21. Kalra, H., Chung, S.Y.K. y Chen, C-J. (1987).- "Phase Equilibrium Data for Supercritical Extraction of Lemon Flavors and Palm Oils with Carbon Dioxide".- Fluid Phase Equilibria 36, 263-278.

22. King, M.B., Bott, T.R., Barr, M.J. y Mahmud, R.S. (1987).- "Equilibrium and Rate Data for the Extraction of Lipids Using Compressed Carbon Dioxide".- Sep. Sci. Technol. 22, 1103-1120.

23. List, G.R. y Friedrich, J.P. (1985).- "Processing Characteristics and Oxidative Stability of Soybean Oil Extracted with Supercritical Carbon Dioxide at $50^{\circ} \mathrm{C}$ and 8000 psi".- J. Am. Oil Chemists' Soc. 62, 82-84.

24. Nilsson, W.B., Gauglitz Jr., E.J. y Hudson, J.K. (1991).- "Solubilities of Methyl Oleate, Oleic Acid, Oleyl Glycerols and Oleyl Glycerol Mixtures in Supercritical Carbon Dioxide".- J. Am. Oil Chemists' Soc. 68, 87-91.

25. Pantinni, Giovanni (1986).- "Estrazione con Fluidi Supercritici (2 parte)".- Tecnol. Chim. 6, 84-89.

26. Perrut, M. (1989).- "Des Fluides Supercritiques: Un Nouveau Type d'Extraction".- BIOFUTUR, Juin, 43-47.

27. Peyron, L. (1984).- "Le Dioxide de Carbone Liquide et Supercritique Solvant d'Extraction des Produits Naturels".- Parfums, Cosmetiques, Aromes 55, 47-53.

28. Prasad, R. (1989).- "Applications of Supercritical Extraction".- CEW 24, 47-49.

29. Rajaraman, K., Narayanan, C.S. y Mathew, A.G. (1984).- "Extraction of Natural Products with Liquid and Super Critical Carbon Dioxide".Indian Food Ind. 3, 48-51.

30. Shishikura, A., Fujimoto, K., Kaneda, T., Arai, K. y Saito, S. (1988)."Concentration of Tocopherols from Soybean Sludge by Supercritical Fluid Extraction".- J. Jpn. Oil Chemists' Soc. (Yukagaku) 37, 8-12.

31. Snyder, J.M., Friedrich, J.P. y Christianson, D.D. (1984).- "Effect of Moisture and Particle Size on the Extractability of Oils from Seeds with Supercritical $\mathrm{CO}_{2}{ }^{\prime \prime} .-\mathrm{J}$. Am. Oil Chemists' Soc. 61, 1851-1856.

32. Stahl, E., Schutz, E. y Mangold, H.K. (1980).- "Extraction of Seed Oils with Liquid and Supercritical Carbon Dioxide".- J. Agric. Food Chem. 28, 1153-1157.

33. Stahl, E., Quirin, K.W. y Totani, N. (1982).- "Extraction et Fractionnement de Lipides et d'autres Produits Naturels à l'Aide de Gaz Supercritiques et Liquéfiés".- Rev. Fr. Corps Gras 6-7, 259-263.

34. Stahl, E., Quirin, K.W. y Gerard, D. (1983). - "Solubilities of Soybean Oil, Jojoba Oil and Cuticular Wax in Dense Carbon Dioxide".- Fett. Seif. Anstrich. 85, 458-463.

35. Tilly, K.D., Chaplin, R.P. y Foster, N.R. (1990).- "Supercritical Fluid Extraction of the Triglycerides Present in Vegetables Oils".- Sep. Sci. Technol. 24, 357-367. 